\title{
Generalized Ward identity and gauge invariance of the color-superconducting gap
}

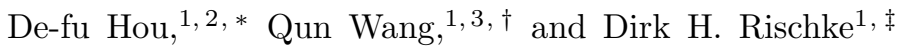 \\ ${ }^{1}$ Institut für Theoretische Physik, J.W. Goethe-Universität, D-60054 Frankfurt/Main, Germany \\ ${ }^{2}$ Institute of Particle Physics, Huazhong Normal University, Wuhan, 430079, P.R. China \\ ${ }^{3}$ Physics Department, Shandong University, Jinan, Shandong, 250100, P.R. China
}

(Dated: November 13, 2018)

\begin{abstract}
We derive a generalized Ward identity for color-superconducting quark matter via the functional integral approach. The identity implies the gauge independence of the color-superconducting gap parameter on the quasi-particle mass shell to subleading order in covariant gauge.
\end{abstract}

PACS numbers: $12.38 . \mathrm{Mh}, 24.85 .+\mathrm{p}$

Due to asymptotic freedom of quantum chromodynamics (QCD) the strong coupling constant $g$ becomes small at large momentum [1]. In cold, dense quark matter, the typical momentum scale is of the order of the quark chemical potential $\mu$. Consequently, at asymptotically large densities, where $\mu \gg \Lambda_{\mathrm{QCD}}$, quark matter is a weakly coupled system. In this case, the dominant interaction between two quarks is single-gluon exchange, which is attractive in the color-antitriplet channel. Due to Cooper's theorem [2], at sufficiently low temperatures the quark Fermi surface becomes unstable with respect to the formation of Cooper pairs. Since this is analogous to what happens in ordinary superconductors [2], this phenomenon was termed color superconductivity [3]. Recently, color superconductivity has been extensively studied within QCD at weak coupling [4, 5, 6, , 7, 8], in phenomenological Nambu-Jona-Lasinio-type models 9, 10], and in effective theories [1, 12, 13, 14, 15].

In a superconductor, exciting particle-hole pairs costs at least an energy amount $2 \phi_{0}$, where $\phi_{0}$ is the value of the superconducting gap parameter at the Fermi surface for zero temperature. The gap parameter can be computed from a gap equation derived in mean-field approximation which involves single-gluon exchange and bare quark-quark-gluon vertices. Schematically, this gap equation can be written in the form [ 6$]$

$$
\phi_{0}=g^{2}\left[\zeta \ln ^{2}\left(\frac{\mu}{\phi_{0}}\right)+\beta \ln \left(\frac{\mu}{\phi_{0}}\right)+\alpha\right] \phi_{0} .
$$

In weak coupling, $g \ll 1$, the solution is $[\underline{5}, 6$, 8 , 16$]$

$$
\phi_{0}=2 b \mu \exp \left(-\frac{c}{g}\right)[1+O(g)] .
$$

The first term in Eq. (11) contains two powers of the logarithm $\ln \left(\mu / \phi_{0}\right)$ : one is the same as in BCS theory [2] (the so-called "BCS logarithm") and the other arises from the collinear exchange of almost static magnetic gluons, which is a long-range interaction [5, [6]. The weak-coupling solution (2) implies that this term contributes to the gap equation at order $O(1)$. We call this term the leading-order term. The value of the coefficient $\zeta$ determines the constant $c$ in Eq. (2). The second term in Eq. (11) contains subleading contributions of order $O(g)$ to the gap equation, characterized by a single power of the logarithm $\ln \left(\mu / \phi_{0}\right) \sim 1 / \mathrm{g}$. A part of them arises from the exchange of non-static magnetic and static electric gluons [6]. Another part is due to the contribution from the regular quark self-energy [17, 18]. Vertex corrections were reported not to contribute to subleading order 18, 19]. The coefficient $\beta$ in Eq. (11) determines the constant $b$ in Eq. (2). The third term in Eq. (11) summarizes sub-subleading contributions of order $O\left(g^{2}\right)$ with neither a collinear nor a BCS logarithm.

In principle, on the quasi-particle mass shell the gap parameter is an observable quantity, and thus must be independent of the choice of gauge. However, the mean-field approximation to the QCD gap equation may violate this requirement. It was argued in Refs. [6, 16, 20] that, in mean-field approximation, gauge-dependent terms enter the QCD gap equation at sub-subleading order. The authors of Ref. 21] confirmed that this is indeed the case for Coulomb gauge and when taking the gap parameter on the quasi-particle mass shell. In contrast to this result, it was pointed out [8, 22] that, in covariant gauge, the gauge-parameter dependence already shows up at subleading order. Denoting the gauge parameter in general covariant gauges by $\xi$, there is then an additional factor $\exp (3 \xi / 2)$ to the prefactor $b$ in Eq. (2).

\footnotetext{
*Electronic address: hou@th.physik.uni-frankfurt.de

${ }^{\dagger}$ Electronic address: qwang@th.physik.uni-frankfurt.de

${ }^{\ddagger}$ Electronic address: drischke@th.physik.uni-frankfurt.de
} 
It is a priori not clear why it should depend on the choice of gauge, at which order gauge-parameter dependent terms appear in the QCD gap equation in mean-field approximation. A posteriori, this result is maybe not that puzzling at all, since the mean-field approximation corresponds to a resummation of a particular class of diagrams which may contribute at different orders for different choices of gauge. In any case, in order to remove the gaugeparameter dependence at subleading order in covariant gauge, one has to take into account corrections to the meanfield approximation. Gauge-parameter dependent terms will then only occur at sub-subleading order. The obvious correction so far not taken into account in the mean-field approximation is to replace bare $q q g$ vertices by full vertices. It is the purpose of this paper to show how the inclusion of vertex corrections guarantees the gauge-parameter independence at subleading order in covariant gauge.

As a matter of fact, it is not even necessary to compute the vertex corrections explicitly. We shall prove the gauge independence in a rather convenient way by making use of a Ward identity which relates the vertex to the inverse propagator. This approach has been frequently applied to show the gauge independence of physical collective excitations in thermal gauge theories, like hot QCD [23, 24]. However, in a superconductor, the existence of a fermion-fermion condensate necessitates the use of the Nambu-Gor'kov (NG) basis to describe the propagation of quasi-particle excitations. It is therefore desirable to derive the Ward identity in the NG basis. In this paper, we derive this generalized Ward identity for color-superconducting quark matter and apply it to the gap equation, thereby showing that the gap parameter is gauge-independent to subleading order on the quasi-particle mass shell.

We note that our approach is similar in spirit to that of Gerhold and Rebhan [25], who used generalized Nielsen identities to give a formal proof that the fermionic quasi-particle dispersion relations in a color superconductor are gauge independent, assuming that the 1PI part of the variation of the effective action with respect to the gauge parameter has no singularities coinciding with those of the quark propagator. Other Ward identities for colorsuperconducting quark matter have been derived by Miransky, Shovkovy, and Wijewardhana [26], but these identities were not suitable to see the gauge independence of the gap parameter to subleading order.

In order to derive the generalized Ward identity, consider QCD with $N_{f}$ quark flavors and quark chemical potential $\mu$. The Lagrangian and the generating functional can be written in the NG basis,

$$
\begin{aligned}
\mathcal{L} & =\frac{1}{2} \bar{\Psi} \mathcal{S}_{0}^{-1} \Psi+\frac{g}{2} \bar{\Psi} \mathcal{T}^{a} \gamma_{\mu} \Psi A_{a}^{\mu}-\frac{1}{4} F_{\mu \nu}^{a} F_{a}^{\mu \nu}-\frac{1}{2 \xi}\left(\partial_{\mu} A_{a}^{\mu}\right)^{2}, \\
Z[J, \bar{H}, H] & =\int[d A][d \bar{\Psi}][d \Psi] \exp \left\{\int_{X}\left[\mathcal{L}+J_{\mu}^{a} A_{a}^{\mu}+\bar{H} \Psi+\bar{\Psi} H\right]\right\} .
\end{aligned}
$$

The quark fields and their sources are given by

$$
\Psi=\left(\begin{array}{c}
\psi \\
\psi_{C}
\end{array}\right), \bar{\Psi}=\left(\bar{\psi}, \bar{\psi}_{C}\right), \quad H=\left(\begin{array}{c}
\eta \\
\eta_{C}
\end{array}\right), \bar{H}=\left(\bar{\eta}, \bar{\eta}_{C}\right)
$$

where the charge conjugate spinors $\psi_{C}, \bar{\psi}_{C}$ are defined through $\psi_{C}=C \bar{\psi}^{T}, \bar{\psi}_{C}=\psi^{T} C$ with the charge conjugation matrix $C=i \gamma^{2} \gamma_{0}$. The inverse free quark propagator (including the chemical potential $\mu$ ) is

$$
\mathcal{S}_{0}^{-1} \equiv\left(\begin{array}{cc}
S_{0,11}^{-1} & 0 \\
0 & S_{0,22}^{-1}
\end{array}\right)
$$

where $S_{0,11}^{-1}=i \gamma^{\mu} \partial_{\mu}+\mu \gamma_{0}-m, S_{0,22}^{-1}=i \gamma^{\mu} \partial_{\mu}-\mu \gamma_{0}-m$. The color part of the qqg vertex is

$$
\mathcal{T}^{a} \equiv\left(\begin{array}{cc}
T^{a} & 0 \\
0 & -T^{a T}
\end{array}\right)
$$

where $T^{a}$ are the generators of $S U(3)_{c}$. The factor $1 / 2$ in front of the first two terms in Eq. (3) accounts for the doubling of the quark degrees of freedom in the NG basis. The gluon field and the field strength tensor are denoted by $A_{\mu}^{a}$ and $F_{\mu \nu}^{a}$, respectively. The last term in $\mathcal{L}$ is the gauge fixing term, and $J_{\mu}^{a}$ is the source term for the gluon field. We denote the space-time integration as $\int_{X} \equiv \int_{0}^{1 / T} d \tau \int_{V} d^{3} \mathbf{x}$. We neglect ghost terms, as ghosts do not contribute to leading and subleading order in cold, dense quark matter [4].

The generating functional $Z$ is invariant under the following infinitesimal $S U(3)_{c}$ gauge transformation

$$
\begin{aligned}
\delta \Psi & =i \theta^{a} \mathcal{T}^{a} \Psi \\
\delta \bar{\Psi} & =-i \theta^{a} \bar{\Psi} \mathcal{T}^{a} \\
\delta A_{\mu}^{a} & =\frac{1}{g} \partial_{\mu} \theta^{a}+f^{a b c} A_{\mu}^{b} \theta^{c} .
\end{aligned}
$$




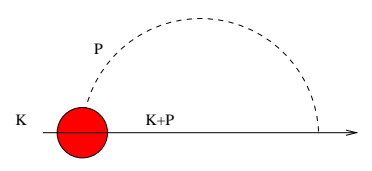

FIG. 1: The quark self-energy in the Dyson-Schwinger equation. The dashed line represents the hard-dense-loop (HDL) dressed propagator, the solid line is the quasi-quark propagator, and the black blob is the full qqg vertex.

The variation of $Z$ with respect to the infinitesimal parameter $\theta^{a}$ of $S U(3)_{c}$ gauge transformations vanishes, which yields the following identity:

$$
-\frac{1}{\xi}\left\{\partial^{2} \partial_{\mu}\left\langle A_{a}^{\mu}\right\rangle+g f^{a b c}\left[\partial_{\mu} \partial_{\nu} D_{b c}^{\mu \nu}(X, X)+\left\langle A_{b}^{\mu}\right\rangle \partial_{\mu} \partial_{\nu}\left\langle A_{c}^{\nu}\right\rangle\right]\right\}-\partial_{\mu} J_{a}^{\mu}+g f^{a b c} J_{\mu}^{b}\left\langle A_{c}^{\mu}\right\rangle+i g\left[\bar{H} \mathcal{T}^{a}\langle\Psi\rangle-\langle\bar{\Psi}\rangle \mathcal{T}^{a} H\right]=0
$$

where the average is taken in the functional sense:

$$
\langle F(A, \bar{\Psi}, \Psi)\rangle=\frac{1}{Z} \int[d A][d \bar{\Psi}][d \Psi] F(A, \bar{\Psi}, \Psi) \exp \left\{\int_{X}\left[\mathcal{L}+J_{\mu}^{a} A_{a}^{\mu}+\bar{H} \Psi+\bar{\Psi} H\right]\right\}
$$

and $D_{b c}^{\mu \nu}=\delta^{2} W / \delta J_{\mu}^{b} \delta J_{\nu}^{c}$ is the two-point Green's function, where $W=\ln Z$ is the generating functional for connected Green's functions. In the hard-dense-loop (HDL) approximation, the two-point function $D_{b c}^{\mu \nu}$ is symmetric in the color indices $b$ and $c$. Consequently, the term proportional to $D_{b c}^{\mu \nu}$ in Eq. (9) vanishes, because the structure constants $f^{a b c}$ multiplying the two-point function are antisymmetric. In a color superconductor this does not need to be true 7], but the violation of symmetry occurs for gluon energies and momenta of order $\sim \phi_{0}$. In the QCD gap equation, this range of gluon energy and momentum contributes only beyond subleading order [].

We can use the effective action $\Gamma[A, \bar{\Psi}, \Psi] \equiv W-\int_{X}\left[J_{\mu}^{a} A_{a}^{\mu}+\bar{H} \Psi+\bar{\Psi} H\right]$ to rewrite the identity (9), i.e., we replace $J_{a}^{\mu}$ by $-\delta \Gamma / \delta A_{\mu}^{a}, \bar{H}$ by $\delta \Gamma / \delta \Psi$, and $H$ by $-\delta \Gamma / \delta \bar{\Psi}$. Note that, from now on, $A_{\mu}^{a}, \bar{\Psi}$, and $\Psi$ denote the expectation values of the gluon and quark fields. Taking the functional derivative $\delta / \delta \Psi\left(X_{1}\right)$ from the right and $\delta / \delta \bar{\Psi}\left(X_{2}\right)$ from the left, and using the fact that the expectation values of the Grassmann-valued quark fields always vanish, $\bar{\Psi}=\Psi \equiv 0$, we obtain the following identity

$$
\begin{aligned}
-\frac{i}{g}\left[\partial_{\mu}^{X} \delta^{a b}-g f^{a b c} A_{\mu}^{c}(X)\right] \frac{\delta^{3} \Gamma[A, 0,0]}{\delta \bar{\Psi}\left(X_{1}\right) \delta A_{\mu}^{b}(X) \delta \Psi\left(X_{2}\right)}= & \delta^{(4)}\left(X-X_{2}\right) \frac{\delta^{2} \Gamma[A, 0,0]}{\delta \bar{\Psi}\left(X_{1}\right) \delta \Psi\left(X_{2}\right)} \mathcal{T}^{a} \\
& -\delta^{(4)}\left(X-X_{1}\right) \mathcal{T}^{a} \frac{\delta^{2} \Gamma[A, 0,0]}{\delta \bar{\Psi}\left(X_{1}\right) \delta \Psi\left(X_{2}\right)} .
\end{aligned}
$$

The expectation value $A_{\mu}^{c}$ of the gluon field on the left-hand side is not necessarily zero in a color superconductor [25, 27, 28]. However, in a two-flavor color superconductor, its value is of order $\phi_{0}^{2} /\left(g^{2} \mu\right)$ [28], i.e., much smaller than the derivative $\partial_{\mu}^{X}$ which is of the order of the gluon energy, $p_{0} \sim \phi_{0}$, or even of the gluon momentum, $p \sim\left(g^{2} \mu^{2} \phi_{0}\right)^{1 / 3}$ [6]. Therefore, in the following we may safely neglect the term proportional to $A_{\mu}^{c}$ on the left-hand side of Eq. (11). We now rewrite Eq. (111) in momentum space,

$$
P_{\mu} \Gamma_{a}^{\mu}(K, P, K+P)=g\left[\mathcal{T}^{a} \mathcal{S}^{-1}(K+P)-\mathcal{S}^{-1}(K) \mathcal{T}^{a}\right],
$$

where $\Gamma_{a}^{\mu}(K, P, K+P)$ is the Fourier transform of $\delta^{3} \Gamma / \delta \bar{\Psi}\left(X_{1}\right) \delta A_{\mu}^{a}(X) \delta \Psi\left(X_{2}\right)$, representing the full $q q g$ vertex, while $\mathcal{S}^{-1}(K)$ is the Fourier transform of $\delta^{2} \Gamma / \delta \bar{\Psi}\left(X_{1}\right) \delta \Psi\left(X_{2}\right)$, i.e., the full inverse quark propagator. We note that the above identity is a $2 \times 2$ matrix identity in the NG basis, and thus a generalized Ward identity. Equation (12) is rather similar to the Ward identity derived by Nambu to prove the gauge invariance of the Meissner effect in ordinary superconductors [29], except that $\mathcal{T}^{a}$ assumes the role of the Pauli matrix $\tau_{3}$ in NG space.

The inverse of the quark propagator $\mathcal{S}^{-1}$ in Eq. (12) is defined as [4]

$$
\mathcal{S}^{-1} \equiv \mathcal{S}_{0}^{-1}+\Sigma,
$$

where $\Sigma$ is the quark self-energy in the NG basis. In momentum space, the Dyson-Schwinger equation for the quark self-energy reads $[4]$

$$
\Sigma(K)=-g \int_{P} \Gamma_{\mu}^{a} \mathcal{S}(K+P) \mathcal{T}^{b} \gamma_{\nu} D_{a b}^{\mu \nu}(P)
$$


The right-hand side of this equation is shown diagrammatically in Fig. 1 Here, $D_{a b}^{\mu \nu}$ is the HDL-resummed gluon propagator and $\int_{P} \equiv T \sum_{n} \int d^{3} \mathbf{p} /(2 \pi)^{3}$, where the sum runs over all fermionic Matsubara frequencies $\omega_{n}=(2 n+$ 1) $\pi T$. The first vertex in the above equation is the full one and the second vertex is the bare one.

We now show with the help of the generalized Ward identity (12) that, on the quasi-particle mass shell, the quark self-energy (14) does not depend on the gauge parameter $\xi$ in covariant gauge. To this end, consider the contribution from the gauge-dependent part of the gluon propagator to the quark self-energy,

$$
\Sigma_{\xi}(K) \equiv g \xi \int_{P} \Gamma_{\mu}^{a} \mathcal{S}(K+P) \mathcal{T}^{a} \gamma_{\nu} \frac{P^{\mu} P^{\nu}}{\left(P^{2}\right)^{2}} .
$$

Using the generalized Ward identity (12), this becomes

$$
\Sigma_{\xi}(K)=g^{2} \xi \int_{P}\left[\mathcal{T}^{a} \mathcal{S}^{-1}(K+P)-\mathcal{S}^{-1}(K) \mathcal{T}^{a}\right] \mathcal{S}(K+P) \mathcal{T}^{a} \gamma_{\nu} \frac{P^{\nu}}{\left(P^{2}\right)^{2}}
$$

Now we put the external momentum $K$ on the quasi-particle mass shell, which is defined as

$$
S^{-1}(K) \Psi_{\text {on-shell }}=0, \quad \bar{\Psi}_{\text {on-shell }} S^{-1}(K)=0,
$$

where $\Psi_{\text {on-shell }}$ is the on-shell quasi-particle wave function. Then, when sandwiching Eq. (16) between on-shell wave functions, the second term in brackets in Eq. (16) vanishes. We arrive at

$$
\Sigma_{\xi}(K)=g^{2} \xi \frac{4}{3} \mathbf{1} \int_{P} \frac{\gamma_{\nu} P^{\nu}}{\left(P^{2}\right)^{2}}=0
$$

where 1 is the $2 \times 2$ unit matrix in the NG basis. The right-hand side vanishes because the integrand of the $P$-integral is an odd function of $P$. Hence, we see that the contribution from the gauge-dependent part of the self-energy (14) vanishes on the quasi-particle mass shell. Therefore, the gap parameter as well as the quasi-particle dispersion relation are gauge-independent in a color superconductor.

This result holds strictly speaking only to subleading order in the QCD gap equation. The reason is that the generalized Ward identity (12) is not an exact identity, but only holds under the following approximations. The first approximation is that we neglected the contribution of ghosts. For QCD, which is a non-Abelian gauge theory, in general one has to take into account ghosts and use the BRST transformation and the Slavnov-Taylor identity or, equivalently, generalized Nielsen identities [23, 25]. However, in dense quark matter, diagrams involving ghosts can be neglected at least to subleading order in the QCD gap equation [4]. The second approximation is that we neglected the contribution from $D_{b c}^{\mu \nu}$ in Eq. (9), arguing that the gluon propagator is symmetric in the color indices. This is true for an HDL-dressed propagator, but not necessarily for the gluon propagator in a color superconductor 7]. However, terms which violate the symmetry of $D_{b c}^{\mu \nu}$ should also be negligible up to subleading order in the gap equation. Finally, the third approximation was to neglect the expectation value of the gluon field in the generalized Ward identity (12). In Ref. [28] it is shown that actually $A_{\mu}^{a} \neq 0$ in order ensure color neutrality. However, at least in a two-flavor color superconductor, $A_{\mu}^{a} \sim \phi_{0}^{2} /\left(g^{2} \mu\right)$, i.e., the expectation value of the gluon field is negligible up to subleading order in the gap equation.

In summary, we derived a generalized Ward identity from QCD for dense, color-superconducting quark matter. The identity implies that, on the quasi-particle mass shell, the gap function and the quasi-particle dispersion relation are independent of the gauge parameter in covariant gauge up to subleading order. There is one potential caveat to this statement. What we have shown is that, to subleading order, the gauge dependence of the quark self-energy (14) arising from the gauge-dependent part of the gluon propagator vanishes on the mass shell. In principle, however, other gauge-dependent terms arise from the gauge dependence of the full vertex $\Gamma_{\mu}^{a}$ when combined with the physical part of the gluon propagator in Eq. (14). In order to show that this is of sub-subleading order in the gap equation, one actually has to compute the gauge-dependent part of the vertex explicitly. This calculation can be simplified considerably with the help of the Ward identity (12), for more details see Ref. [30]. The result is that also this contribution is of sub-subleading order in the gap equation.

Our result shows that in order to obtain a gauge-independent gap function up to subleading order, one has to use the full vertex as well as the full fermion propagator in the NG basis. A consequence is that the prefactor $\exp (3 \xi / 2)$ to the gap function found in the mean-field approximation [8, 22] will be removed by contributions from the full $q q g$ vertex when taking the gap function on the quasi-particle mass shell. An explicit diagrammatic proof of this statement will be presented elsewhere [30]. 


\section{Acknowledgments}

The authors thank R. Pisarski, A. Rebhan, H.-c. Ren, and I. Shovkovy for interesting discussions and a critical reading of the manuscript. D. Hou acknowledges financial support from the Alexander von Humboldt-Foundation and the National Natural Science Foundation of China under grants 1000502 and 10135030. He also appreciates help and support from the Institut für Theoretische Physik of the J.W. Goethe-Universität, and especially from Prof. W. Greiner. The work of Q. Wang is supported by GSI Darmstadt and BMBF.

[1] D.J. Gross and F. Wilczek, Phys. Rev. Lett. 30, 1343 (1973); H.D. Politzer, ibid. 1346.

[2] J. Bardeen, L.N. Cooper, and J.R. Schrieffer, Phys. Rev. 108, 1175 (1957); M. Tinkham, Superconductivity (Gordon and Breach, Science Publishers, New York, 1965).

[3] D. Bailin and A. Love, Phys. Rep. 107, 325 (1984).

[4] for a review, see e.g.: D.H. Rischke, nucl-th/0305030

[5] D.T. Son, Phys. Rev. D 59, 094019 (1999).

[6] R.D. Pisarski and D.H. Rischke, Phys. Rev. D 61, 051501, 074017 (2000); A. Schmitt, Q. Wang, and D.H. Rischke, Phys. Rev. D 66, 114010 (2002); nucl-th/0301090

[7] D.H. Rischke, Phys. Rev. D 62, 054017 (2000); Phys. Rev. D 64, 094003 (2001);

[8] D.K. Hong, V.A. Miransky, I.A. Shovkovy, and L.C.R. Wijewardhana, Phys. Rev. D 61, 056001 (2000); Erratum, 62, 059903 (2000); I.A. Shovkovy, L.C.R. Wijewardhana, Phys. Lett. B 470, 189 (1999).

[9] M. Alford, K. Rajagopal, and F. Wilczek, Phys. Lett. B 422, 247 (1998); R. Rapp, T. Schäfer, E.V. Shuryak, and M. Velkovsky, Phys. Rev. Lett. 81, 53 (1998).

[10] I.A. Shovkovy and M. Huang, Phys. Lett. B 564, 205 (2003). F. Neumann, M. Buballa, and M. Oertel, Nucl. Phys. A714, 481 (2003); M. Buballa, J. Hosek, and M. Oertel, Phys. Rev. Lett. 90, 182002 (2003); A. Mishra and H. Mishra, hep-ph/0306105

[11] D.T. Son and M.A. Stephanov, Phys. Rev. D 61, 074012 (2000).

[12] D.K. Hong, Nucl. Phys. B582, 451 (2000); Phys. Lett. B 473, 118 (2000).

[13] R. Casalbuoni and R. Gatto, Phys. Lett. B 464, 111 (1999).

[14] T. Schäfer, Nucl. Phys. A715, 879 (2003); Phys. Rev. D 65, 074006 (2002); Nucl. Phys. A728, 251 (2003); P.F. Bedaque and T. Schäfer, Nucl. Phys. A697, 802 (2002);

[15] K. Iida and G. Baym, Phys. Rev. D 63, 074018 (2001), Erratum-ibid. 66, 059903 (2002); I. Giannakis and H.-c. Ren, Phys. Rev. D 65, 054017 (2002); Nucl. Phys. B669, 462 (2003).

[16] T. Schäfer and F. Wilczek, Phys. Rev. D 60, 114033 (1999).

[17] Q. Wang and D.H. Rischke, Phys. Rev. D 65, 054005 (2002).

[18] W.E. Brown, J.T. Liu, and H.-c. Ren, Phys. Rev. D 61, 114012 (2000); 62, 054013 (2000); 62, 054016 (2000).

[19] Q. Wang, A. Mishra, and D.H. Rischke, in preparation.

[20] K. Rajagopal and E. Shuster, Phys. Rev. D 62, 085007 (2000).

[21] R.D. Pisarski and D.H. Rischke, Nucl. Phys. A702, 177 (2002).

[22] D.K. Hong, T. Lee, D.-P. Min, D. Seo, and C. Song, Phys. Lett. B 565, 153 (2003).

[23] R. Kobes, G. Kunstatter, and A. Rebhan, Phys. Rev. Lett. 64, 2992 (1990); Nucl. Phys. B355, 1 (1991); A. Rebhan, Lect. Notes Phys. 583, 161-208(2002).

[24] E. Braaten and R.D. Pisarski, Phys. Rev. D 42, 2156 (1990).

[25] A. Gerhold and A. Rebhan, Phys. Rev. D 68, R011502 (2003).

[26] V.A. Miransky, I.A. Shovkovy, and L.C.R. Wijewardhana, Phys. Rev. D 62, 085025 (2000); 63, 056005 (2001).

[27] A. Kryjevski, Phys. Rev. D 68, 074008 (2003).

[28] D.D. Dietrich and D.H. Rischke, nucl-th/0312044

[29] Y. Nambu, Phys. Rev. 117, 648 (1960).

[30] D. Hou, Q. Wang, and D.H. Rischke, in preparation. 